\title{
INFLUÊNCIA DOS FATORES METEOROLÓGICOS E FLORA APÍCOLA SOBRE O PESO DE COLMEIAS DE Apis mellifera L. NO SERTÃO PERNAMBUCANO
}

\section{INFLUENCE OF METEOROLOGICAL FACTORS AND BEEKEEPING FLORA ON THE WEIGHT OF BEEHIVES OF Apis mellifera L. IN THE SERTÃO PERNAMBUCANO}

\author{
Pedro de Assis de Oliveira ${ }^{1}$; Catriel Henrique Edgard Dantas Cardozo ${ }^{2}$; Yan Marcelo Neves \\ dos Anjos ${ }^{3}$; Marcelo Casimiro Cavalcante ${ }^{4}$; Marcelo de Oliveira Milfont ${ }^{5}$
}

DOI: https://doi.org/10.31692/978-65-991061-7-0.175-182

\section{RESUMO}

O presente trabalho teve como objetivo avaliar a influência da precipitação acumulada, umidade relativa do ar, temperatura do ar e flora apícola, sobre o peso de ninhos e melgueiras de colônias povoadas por abelhas Apis mellifera, na região do Sertão pernambucano. O experimento foi conduzido no apiário da Universidade Federal Rural de Pernambuco Unidade Acadêmica de Serra Talhada, entre os meses de março a junho de 2018. Um total de 20 colmeias foi utilizado para a pesagem dos ninhos e melgueiras. Paralelamente, foi realizado o levantamento da flora apícola e coletados dados diários da precipitação acumulada $(\mathrm{mm})$, umidade relativa do ar $(\%)$ e temperatura do ar $\left({ }^{\circ} \mathrm{C}\right)$ através do site do Instituto Nacional de Meteorologia-INMET, onde a estação automática está localizada a 400 metros do apiário. Regressões entre os dados foram estabelecidas. O peso médio dos ninhos foi de $21,44 \mathrm{Kg} \pm 3,09$ (DP) e das melgueiras $6,90 \mathrm{Kg} \pm 2,34$ (DP). Houve relação significativa da precipitação com o peso do ninho e da melgueira sendo $67 \%$ e $97 \%$ respectivamente, com maior influência no peso das melgueiras do que o ninho. A umidade relativa do ar, o coeficiente de determinação foi mais alto para o ninho e menor para a melgueira com $96 \%$ e $50 \%$ concomitantemente. Em relação à temperatura do ar o coeficiente de determinação explica $99 \%$, em relação ao peso do ninho. Já no peso da melgueira é de $57 \%$. Houve relação significativa entre o número de plantas apícolas em floração com o peso do ninho e a melgueira com $99 \%$ e $37 \%$ simultaneamente. Os parâmetros meteorológicos, especialmente a precipitação, atuam diretamente sobre as floradas e, consequentemente, sobre $\mathrm{o}$ desenvolvimento e a produção de mel de colônias abelhas Apis mellifera.

Palavras-Chave: Africanizada, colônia, mel, produção.

\section{ABSTRACT}

The objective of this work was to evaluate the influence of accumulated precipitation, relative air humidity, air temperature and bee flora on the weight of nests and mound colonies populated by Apis mellifera bees in the Sertão region of Pernambuco. The experiment was conducted in the apiary of the Federal Rural University of Pernambuco - Academic Unit of

\footnotetext{
${ }^{1}$ Zootecnista, Mestrando em Ciência Animal e Pastagens, Universidade Federal Rural de Pernambuco- Unidade Acadêmica de Garanhuns, Bolsista da Coordenação de Aperfeiçoamento de Pessoal de Nível Superior- CAPES. E-mail: pedromanari@hotmail.com.

${ }^{2}$ Bacharelando em Zootecnia, Universidade Federal Rural de Pernambuco- Unidade Acadêmica de Serra Talhada, E-mail: catrielhenrique.dantas@gmail.com.

${ }^{3}$ Bacharelando em Agronomia, Universidade Federal Rural de Pernambuco- Unidade Acadêmica de Serra Talhada, E-mail: yan_scc@hotmail.com.

${ }^{4}$ Zootecnista, Doutor em Zootecnia, Universidade Federal Rural de Pernambuco - Unidade Acadêmica de Serra Talhada, E-mail: marcelufc@yahoo.com.br.

${ }^{5}$ Agrônomo, Doutor em Zootecnia, Universidade Federal Rural de Pernambuco - Unidade Acadêmica de Garanhuns, E-mail: marcelo_m_agro@yahoo.com.br.
} 
Serra Talhada, between March and June 2018. A total of 20 hives were used to weigh nests and menders. At the same time, the apicultural flora was collected and daily data of accumulated precipitation $(\mathrm{mm})$, relative humidity $(\%)$ and air temperature $\left({ }^{\circ} \mathrm{C}\right)$ were collected through the National Institute of Meteorology (INMET) is located 400 meters from the apiary. Regressions between the data were established. The mean weight of the nests was $21.44 \mathrm{~kg} \pm 3.09$ (SD) and the mound weight $6.90 \mathrm{~kg} \pm 2.34(\mathrm{SD})$. There was a significant relationship between rainfall and nest weight, with $67 \%$ and $97 \%$ respectively, with a greater influence on the weight of the mussels than the nest. The relative air humidity, the coefficient of determination was higher for the nest and lower for the melgueira with $96 \%$ and $50 \%$ concomitantly. In relation to the air temperature the coefficient of determination explains $99 \%$, in relation to the weight of the nest. Already in the weight of the melgueira is of $57 \%$. There was a significant correlation between the number of apiculture plants in flowering with nest weight and the melgueira with $99 \%$ and $37 \%$ simultaneously. The meteorological parameters, especially the precipitation, act directly on the flowering ones and, consequently, on the development and the production of honey of bee colonies Apis mellifera.

Keywords: Africanized, colony, honey, production.

\section{INTRODUÇÃO}

A apicultura, criação de abelhas do gênero Apis vem crescendo cada vez mais nas Américas, com destaque para o Brasil por possuir um híbrido conhecido como africanizadas. Abelhas produtivas, altamente adaptadas às condições edafoclimáticas do país e extremamente resistentes a várias pragas e doenças que acometem a atividade no restante do mundo (WINSTON, 2003).

Os fatores bióticos (florescimento das plantas) e abióticos (elementos climáticos: precipitação, temperatura do ar, umidade relativa do ar, radiação solar, nebulosidade e ventos), interferem no desenvolvimento das crias, no desenvolvimento da colônia, e consequentemente na produção dos diversos produtos apícolas (mel, pólen, cera, geleia real, própolis e apitoxina). (SOUZA et al., 2011).

Para o êxito da atividade é de grande relevância que o apicultor tenha conhecimentos da oferta dos recursos tróficos por parte das plantas, bem como, o entendimento das variáveis meteorológicas sobre a produção das colmeias. Essas informações servem de subsídio ao produtor para a adoção de práticas de manejos corretas (PEREIRA et al., 2006; SANTOS; KIILL e ARAÚJO, 2006).

São escassas as informações disponíveis na literatura da influência dos fatores meteorológicos e flora apícola sobre a flutuação do peso e desenvolvimento de colônias de abelhas melíferas, principalmente nas condições do Semiárido brasileiro.

Assim, objetivou-se avaliar a influência da precipitação acumulada, umidade relativa do ar, temperatura e do pasto apícola sobre o peso de ninhos e melgueiras de colmeias povoadas por abelhas Apis mellifera, no estado de Pernambucano. 


\section{FUNDAMENTAÇÃO TEÓRICA}

O Semiárido brasileiro proporciona condições ideais para a criação de abelhas africanizadas (Apis mellifera) (KHAN; MATOS e LIMA, 2009). De acordo com Costa et al. (2007) a reprodução e produção de uma colônia são influenciadas pelas variáveis climáticas somadas a disponibilidade do pasto apícola. O Bioma Caatinga tem uma grande diversidade de flora apícola no período chuvoso reduzindo no período mais seco do ano, fazendo com que a produção de mel acompanhe essa sazonalidade (PEREIRA et al., 2006).

Ocorre, também, uma variação na flora de acordo com as localidades ou zonas, influenciando o fluxo de recursos polínicos e nectaríferos que entra na colônia e, consequentemente, na composição do mel (BENEVIDES e CARVALHO, 2012; LOPES et al., 2016).

O crescimento e produção de uma colônia são dependentes, também, das práticas de manejo realizadas. O simples fato de abrir uma colônia para inspeções e revisões afeta diretamente na capacidade de termorregulação das colônias, de forma que as abelhas direcionam mais tempo para manter a zona de conforto do ambiente, gastando energia que poderia ser investida nas atividades de desenvolvimento da colônia (WINSTON, 2003; SEELEY, 2006; BRASIL et al., 2013). Sendo assim, as pesagens das colmeias poderiam funcionar como uma estratégia de acompanhamento do desenvolvimento, reduzindo a abertura durante as revisões.

\section{METODOLOGIA}

O presente trabalho foi conduzido no apiário da Unidade Acadêmica de Serra Talhada-UAST, Universidade Federal Rural de Pernambuco-UFRPE, durante os meses de março a junho de 2018. Foram utilizadas 20 colônias de Apis mellifera bem adaptadas à região, previamente homogeneizadas em relação à quantidade de crias e alimento, e seus pesos registrados (ninho e melgueira). Para avaliação do desenvolvimento das colmeias foi realizada a pesagem uma vez por mês, pesando, separadamente, as melgueiras (deposição de mel) e os ninhos (crescimento da colônia) (Figura 1). Ao final, os dados médios foram obtidos e os desvios padrões (DP) calculados. Quanto ao manejo, foram realizadas revisões quinzenais conforme preconizadas por práticas apícolas (SENAR, 2010a).

No mesmo momento das revisões e pesagens, realizava-se o levantamento da flora apícola adaptado do SENAR (2010b), através da coleta de material botânico fértil. Essas coletas partiam do apiário como ponto central nas quatro direções (norte, sul, leste, oeste), em transectos aleatórios de cerca de 1.500m de comprimento, entre 7:00 e 17:00 horas. 
Foram coletados os ramos férteis das plantas apícolas com auxílio de tesoura de poda. Em seguida, os ramos foram prensados entre grades de madeira, papelões, folhas de jornal e placas de alumínio corrugado, e posteriormente, desidratado em estufa de lâmpada, conforme Mori et al. (1989). As exsicatas foram depositadas no Herbário do Semiárido do Brasil (HESBRA) da UFRPE/UAST. As identificações das plantas foram baseadas em bibliografia especializada ou por comparação com material do acervo do referido herbário sobre a orientação de especialista.

Dados diários da precipitação acumulada $(\mathrm{mm})$, umidade relativa do ar $(\%)$, temperatura do ar $\left({ }^{\circ} \mathrm{C}\right)$ foram adquiridos através do site do Instituto Nacional de Meteorologia-INMET, Onde a estação automática está localizada a 400 metros do apiário. Os dados em escala diária foram convertidos para mensal. Regressões, utilizando-se o Programa Computacional Microsoft Excel foram estabelecidas entre a temperatura, umidade, precipitação e flora apícola sobre o peso das melgueiras e ninhos em escalas mensais.

Figura 1- Pesagens das colmeias durante o experimento no município em Serra Talhada-PE, 2018: a) pesagem do ninho (com fundo e tampa); b) pesagem da melgueira.

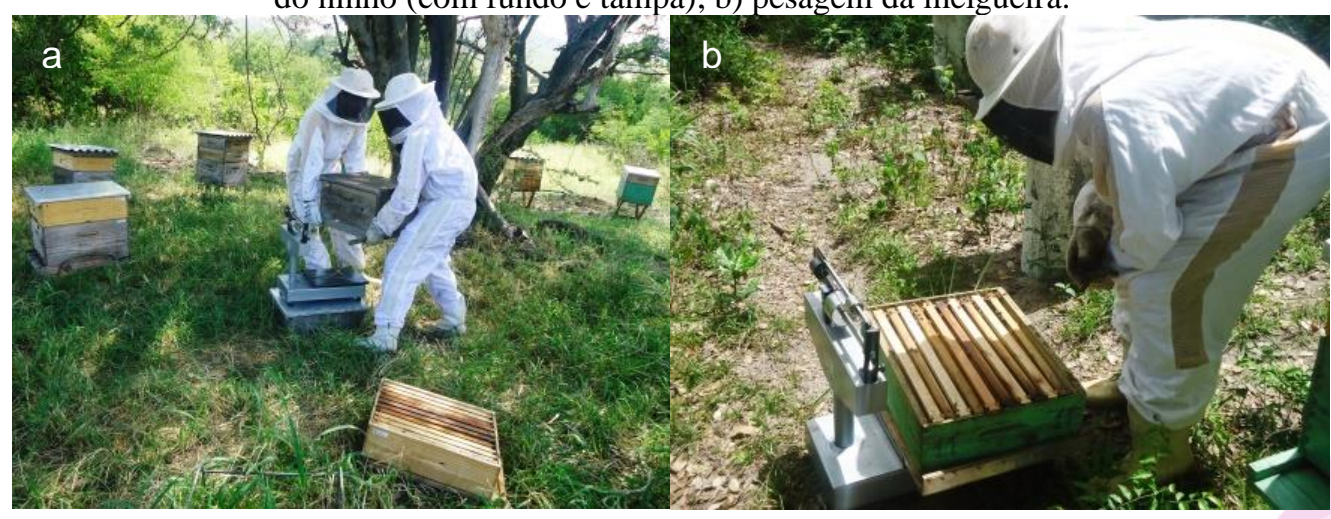

\section{RESULTADOS E DISCUSSÃO}

Durante o período do experimento, o peso do ninho apresentou pequenas variações, com exceção no mês de junho, onde foi registrado $19,00 \mathrm{~kg} \pm 2,48$ (DP). Em relação às melgueiras, verificou-se um acréscimo de mel acumulado, ocasionando ganho de peso. Inicialmente, apresentavam $5,00 \mathrm{~kg} \pm 0.28$ (DP) e em junho, final do experimento atingiram $8,00 \mathrm{~kg} \pm 2.08(\mathrm{DP})$.

O período de maior precipitação acumulado foi o primeiro mês de experimento, reduzindo a cada mês. A curva do peso dos ninhos, que indica o desenvolvimento da colônia, acompanhou essa redução das chuvas, porém de forma bem suave. Contrariamente, o peso das melgueiras aumentou gradativamente (Figura 2). As floradas tiveram também um crescimento, até o mês de abril, quando iniciou uma redução. O comportamento das floradas responde às precipitações, uma vez que as chuvas são fontes de estímulos reprodutivos a 
muitas espécies apícolas, especialmente aquelas de extrato herbáceo e arbustivo (SANTOS; KIILL e ARAÚJO, 2006).

Algumas espécies respondem mais rapidamente às chuvas e outras mais tardiamente, justificando a permanência de muitas espécies em florescimento mesmo com baixos índices pluviométricos (WOLFF et al., 2006; OLIVEIRA et al, 2017). As abelhas, por sua vez, responderam a esses florescimentos coletando néctar e acumulando mel nas melgueiras. $\mathrm{O}$ peso dos ninhos permaneceu praticamente constante, já que as operárias mantiveram fluxo de alimento sem muitas oscilações e a rainha manteve a postura em resposta a esse comportamento.

Figura 2- Peso médio do ninho e melgueira $(\mathrm{Kg})$ de colônias povoadas com abelhas africanizadas (Apis mellifera) e dados de precipitação acumulada $(\mathrm{mm})$ e número de plantas apícolas em florescimento durante o período de março a junho de 2018, no município de Serra Talhada-PE.

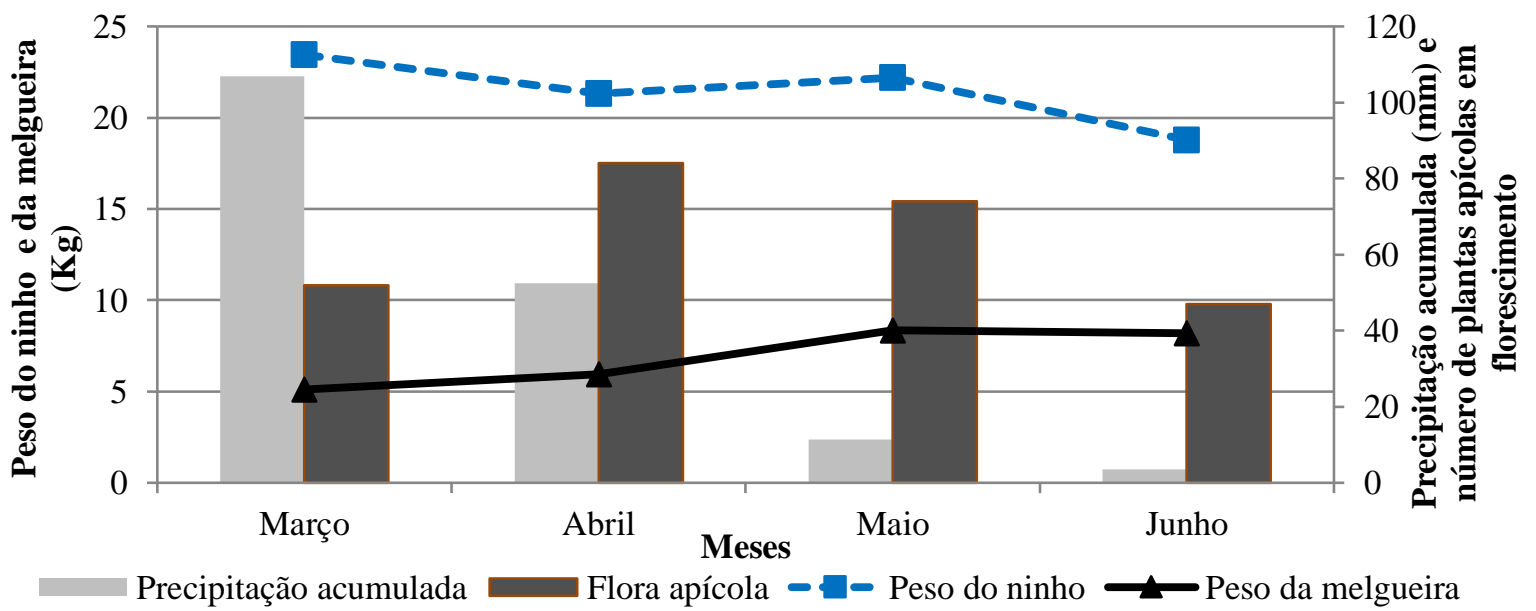

Houve relação significativa entre a precipitação com o peso do ninho e com a melgueira sendo $67 \%$ e $97 \%$, respectivamente (Figura 3 A/B), indicando que a precipitação influenciou mais sobre o peso das melgueiras do que o ninho. Já pra umidade relativa do ar o coeficiente de determinação foi mais alto para o ninho e menor para a melgueira com $96 \%$ e $50 \%$, concomitantemente (Figura $3 \mathrm{C} / \mathrm{D}$ ). Em relação à temperatura do ar externa à colmeia o coeficiente de determinação explica $99 \%$, em relação ao peso do ninho. Já no peso da melgueira é de $57 \%$ (Figura 3 F/E). 
Figura 3-Regressão da precipitação acumulada, umidade relativa do ar e temperatura do ar com o peso da melgueira e do ninho em Serra Talhada-PE, 2018.
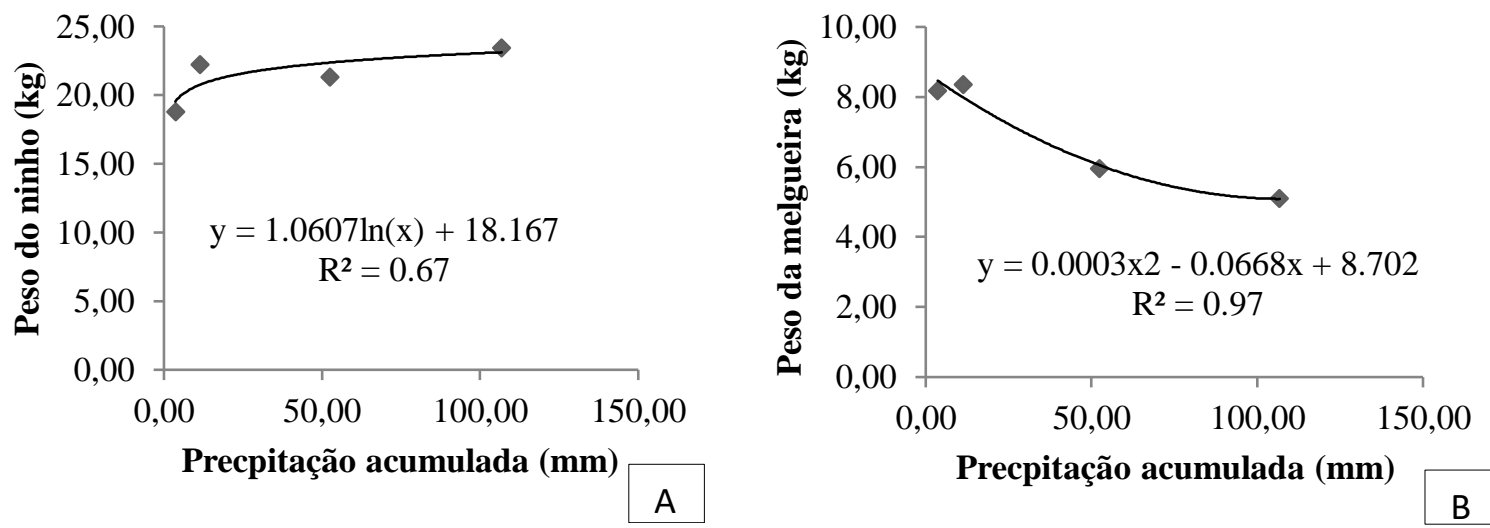

Precpitação acumulada $(\mathbf{m m})$ B
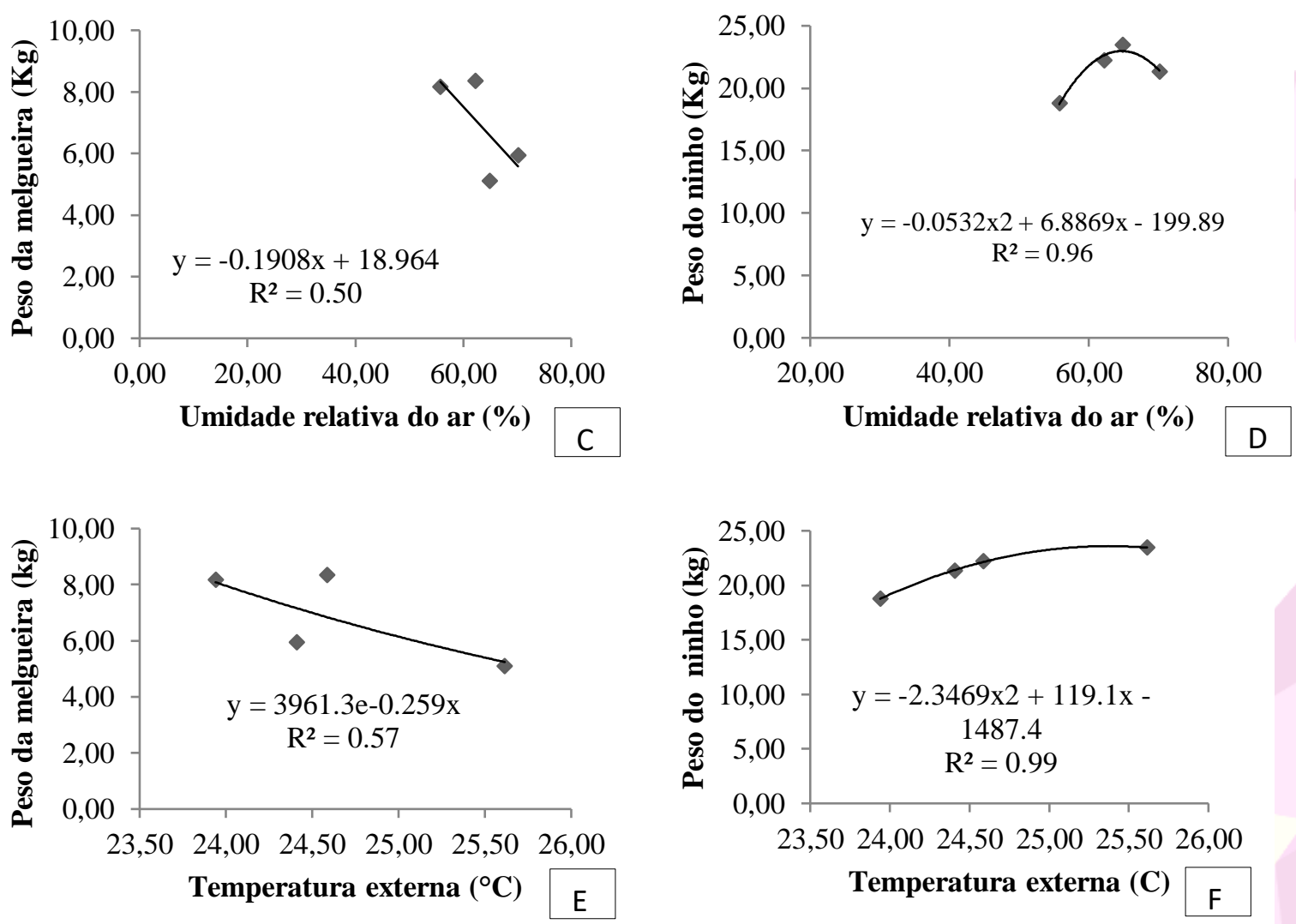

Houve relação expressiva entre o número de plantas apícolas em floração com o peso da colônia (ninho com melgueira), sendo 99\%. Indicando que a flora apícola influenciou de forma decisiva sobre o peso das colônias (Figura 4). O qual a precipitação e essencial para o florescimento das plantas e consequentemente aumenta o forrageamento por parte das abelhas campeiras para coleta dos recursos (néctar, pólen e resina). Desta forma, o conhecimento do calendário de flora apícola de uma localidade é essencial para se planejar a produção apícola. 
Figura 4- Regressão do número de plantas em floração, com o peso da colônia (ninho com melgueira) em Serra Talhada-PE, 2018.

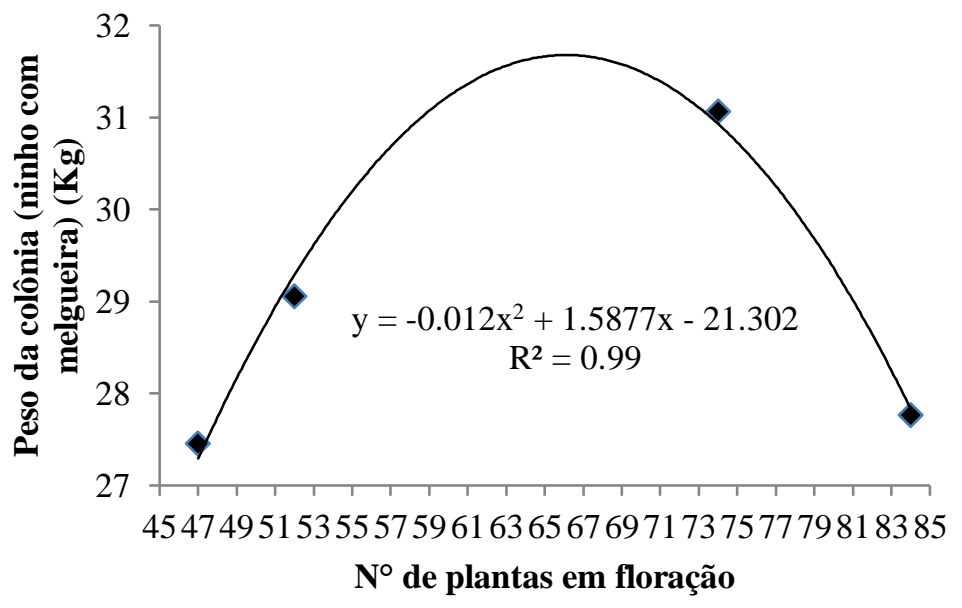

\section{CONCLUSÕES}

Os parâmetros meteorológicos, especialmente a precipitação, atuam diretamente sobre as floradas e, consequentemente, sobre o desenvolvimento e a produção de mel de colônias de abelhas Apis mellifera.

\section{REFERÊNCIAS}

BENEVIDES, D. S.; CARVALHO, F.G. Levantamento da flora apícola presente em áreas de caatinga do município de Caraúbas-RN. Sociedade e Território, v. 21, n. 1 e 2, p. 44-54, 2012.

BRASIL, D. F.; GUIMARÃES, M. O. ; BARBOSA FILHO, J. A. D. ; FREITAS, B. M. internal ambience of bee colonies submitted to strengthening management by adding broods. Engenharia Agrícola, v. 34, n. 5, p. 902-909, 2013.

COSTA, F. M. et al. Desenvolvimento de colônias de abelhas Apis mellifera africanizadas na região de Maringá, Estado do Paraná. Acta Scientiarum. Animal Sciences, v. 29, n. 1, p. 101-108, 2007.

KHAN, A. S.; MATOS, V. D.; LIMA, P. V. P. S. Desempenho da apicultura no estado do Ceará: competitividade, nível tecnológico e fatores condicionantes. Revista de Economia e Sociologia Rural, v. 47, n. 3, p. 651-676, 2009.

LOPES, C. G. R.; BEIRÃO, D. C. C.; PEREIRA, L. A.; ALENCAR, L. C. Levantamento da flora apícola em área de cerrado no município de Floriano, estado do Piauí, Brasil. Revista Brasileira de Biociências, v. 14, n. 2, 2016.

MORI, S. A; SILVA, L. A. M.; LISBOA, G. \& CORADIN, L. 1989. Manual de manejo do herbário fanerogâmico. CEPLAC, Ilhéus.

OLIVEIRA, P. A.; SA, M. S.; MILFONT, M. O. ; MELO, A. L.; CAVALCANTE, M. C. . 
Flora apícola em área de Caatinga, Serra Talhada-PE. In: Anais......II Congresso Internacional das Ciências Agrárias- COINTER PDVAGRO, 2017, Natal-RN. Desenvolvimento do Campo: a Ciência e Tecnologia a Serviço da Sustentabilidade, 2017.

PEREIRA, F.M.; FREITAS, B.M.; VIEIRA-NETO, J.M.; LOPES,M.T.R.; BARBOSA, A.L.; CAMARGO, R.C.R. Desenvolvimentode colônias de abelhas com diferentes alimentos protéicos. Pesquisa Agropecuária Brasileira, v.41, p.1-7, 2006.

SANTOS, R. F.; KIILL, L. H. P.; ARAÚJO, J. L. P. Levantamento da flora melífera de interesse apícola no município de Petrolina-PE. Revista Caatinga, v. 19, n. 3, 2006.

SEELEY, T. D. Ecologia da Abelha: um estudo de adaptação na vida social (tradução de C.A. Osowski). Porto Alegre: Paixão Editores LTDA, 2006. 256p.

SENAR- Serviço Nacional de Aprendizagem Rural. Mel: manejo de apiário para produção do mel. Brasília: Coleção SENAR 142. 2. ed. 2010a. 80 p.

SENAR- Serviço Nacional de Aprendizagem Rural. Abelhas Apis mellífera: Instalação do apiário. Brasília: Coleção SENAR 141. 2. ed. 2010b. 80 p.

SOUZA, D.N.; EVANGELISTA-RODRIGUES, A.; CRUZ, G.R.B.; RODRIGUES, M.L.; SILVA, M.C. \& GOIS, G.C. Modelos matemáticos aplicados no crescimento de colméias de Apis mellifera L. no Cariri Paraibano. Archivos de zootecnia, v. 60, n. 229, p. 137-140, 2011.

WINSTON, M. L. A biologia da abelha. (tradução de C.A. Osowski). Porto Alegre: Ed. Magister, 2003. 276 p.

WOLFF, L. F., LOPES, M. D. R., PEREIRA, F. D. M., CAMARGO, R. C. R., \& NETO, J. M. V. (2006). Localização do apiário e instalação das colméias. Embrapa Meio-Norte. 